\title{
Johnson Noise Thermometry for Advanced Small Modular Reactors
}

\section{September 2012}

Prepared by

Charles L. Britton Jr.

Michael Roberts

Nora D. Bull

David E. Holcomb

Richard T. Wood

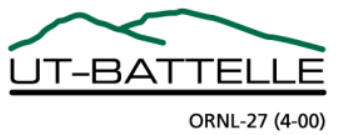




\title{
DOCUMENT AVAILABILITY
}

Reports produced after January 1, 1996, are generally available free via the U.S. Department of Energy (DOE) Information Bridge.

Web site http://www.osti.gov/bridge

Reports produced before January 1, 1996, may be purchased by members of the public from the following source.

\author{
National Technical Information Service \\ 5285 Port Royal Road \\ Springfield, VA 22161 \\ Telephone 703-605-6000 (1-800-553-6847) \\ TDD 703-487-4639 \\ Fax 703-605-6900 \\ E-mail info@ntis.gov \\ Web site http://www.ntis.gov/support/ordernowabout.htm
}

Reports are available to DOE employees, DOE contractors, Energy Technology Data Exchange (ETDE) representatives, and International Nuclear Information System (INIS) representatives from the following source.

Office of Scientific and Technical Information

P.O. Box 62

Oak Ridge, TN 37831

Telephone 865-576-8401

Fax 865-576-5728

E-mail reports@osti.gov

Web site http://www.osti.gov/contact.html

This report was prepared as an account of work sponsored by an agency of the United States Government. Neither the United States Government nor any agency thereof, nor any of their employees, makes any warranty, express or implied, or assumes any legal liability or responsibility for the accuracy, completeness, or usefulness of any information, apparatus, product, or process disclosed, or represents that its use would not infringe privately owned rights. Reference herein to any specific commercial product, process, or service by trade name, trademark, manufacturer, or otherwise, does not necessarily constitute or imply its endorsement, recommendation, or favoring by the United States Government or any agency thereof. The views and opinions of authors expressed herein do not necessarily state or reflect those of the United States Government or any agency thereof. 
ORNL/TM-2012/346

SMR/ICHMI/ORNL/TR-2012/01

\title{
JOHNSON NOISE THERMOMETRY FOR ADVANCED SMALL MODULAR REACTORS
}

\author{
Author(s) \\ Charles L. Britton Jr \\ Michael Roberts \\ Nora D. Bull \\ David E. Holcomb \\ Richard T. Wood
}

Date Published: September 2012

\author{
Prepared by \\ OAK RIDGE NATIONAL LABORATORY \\ Oak Ridge, Tennessee 37831-6283 \\ managed by \\ UT-BATTELLE, LLC \\ for the \\ U.S. DEPARTMENT OF ENERGY \\ under contract DE-AC05-00OR22725
}




\section{CONTENTS}

Page

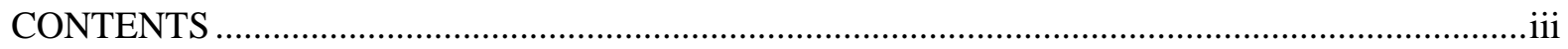

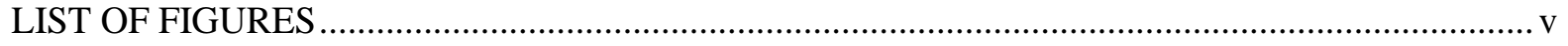

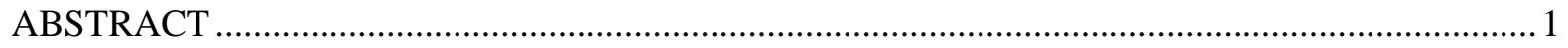

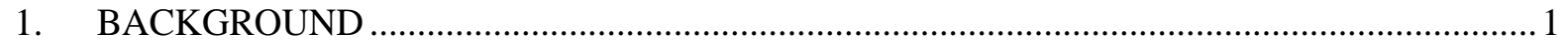

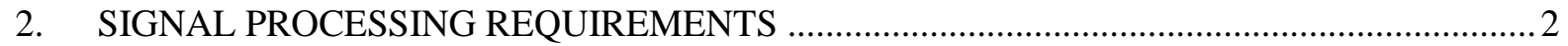

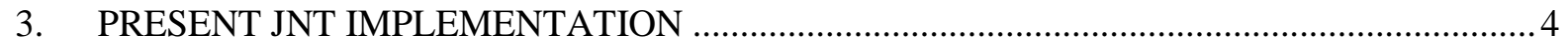

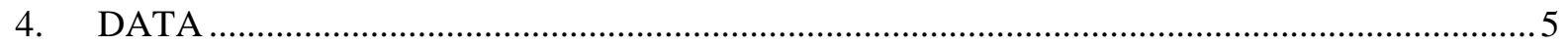

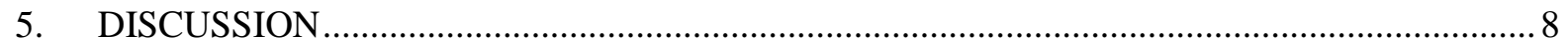

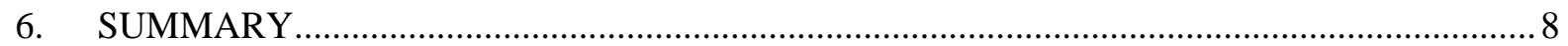

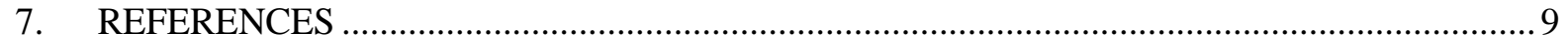




\section{LIST OF FIGURES}

Figure

Page

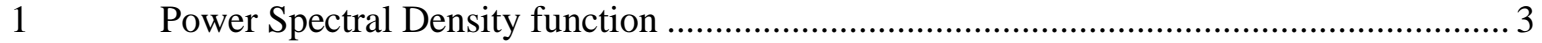

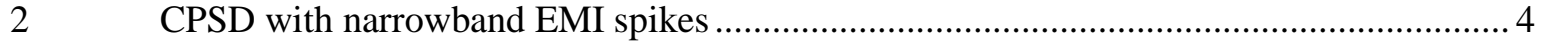

3 Johnson Noise Thermometry measurement process schematic ...........................5

$4 \quad$ Principal components and conceptual configuration ......................................6

$5 \quad$ Representative screen from the JNT measurement software ................................. 


\begin{abstract}
Temperature is a key process variable at any nuclear power plant (NPP). The harsh reactor environment causes all sensor properties to drift over time. At the higher temperatures of advanced NPPs the drift occurs more rapidly. The allowable reactor operating temperature must be reduced by the amount of the potential measurement error to assure adequate margin to material damage. Johnson noise is a fundamental expression of temperature and as such is immune to drift in a sensor's physical condition. In and near the core, only Johnson noise thermometry (JNT) and radiation pyrometry offer the possibility for long-term, high-accuracy temperature measurement due to their fundamental natures. Small Modular Reactors (SMRs) place a higher value on long-term stability in their temperature measurements in that they produce less power per reactor core and thus cannot afford as much instrument recalibration labor as their larger brethren. The purpose of the current ORNL-led project, conducted under the Instrumentation, Controls, and Human-Machine Interface (ICHMI) research pathway of the U.S. Department of Energy (DOE) Advanced SMR Research and Development (R\&D) program, is to develop and demonstrate a drift free Johnson noise-based thermometer suitable for deployment near core in advanced SMR plants.
\end{abstract}

\title{
1. BACKGROUND
}

Reliable measurement of reactor core temperature is necessary for control of reactor power output and maintaining a safe thermal margin to core damage. JNT was demonstrated for in-core temperature measurement thirty years ago [1] and more generally, Johnson noise was demonstrated for temperature measurement more than fifty years ago [2]. An overview of the application of Johnson noise to high-temperature reactors was published in 2005[3] but despite the decades of admittedly intermittent effort, no Johnson noise (or radiation pyrometry[4]) instrument has yet reached the commercial market. The underlying reason for this is that Johnson noise is a small-signal phenomenon. Measuring the Johnson noise in isolation from all of the additional electrical signals that inevitably occur in a plant deployment is technically daunting. This project is relying on the characteristic shape of the Johnson noise power spectrum, combined with recent advances in digital signal processing as well as progressive improvements in the measurement electronics design required to extract the Johnson noise signal.

Johnson noise is a first-principles representation of temperature. Fundamentally, temperature is merely a convenient representation of the mean translational kinetic energy of an atomic ensemble. Since Johnson noise is a fundamental representation of temperature rather than a response to temperature such as electrical resistance or thermoelectric potential, Johnson noise is immune from chemical and mechanical changes in the material properties of the sensor. One consequent advantage of JNT is that the actual resistive element is not required to follow a particular temperature versus resistance curve. This allows consideration of high stability, high mechanical strength alloys or even a cermet element as the temperature transducer-likely significantly increasing the lifetime and reliability of the sensor element. While the JNT amplifier electronics restrict the allowable range of transducer resistance shift with temperature to roughly a factor of three around an operational resistance of tens or hundreds of ohms, the exact shape of the resistance-to-temperature correspondence is not otherwise constrained. 
JNT is best understood as a continuous, first-principles re-calibration methodology for a conventional resistance-based temperature measurement technique. The traditional method of directly measuring temperature from a resistance temperature detector (RTD) has unavoidable, unacceptable drift. JNT measurement is applied in parallel to the RTD lead wires of the resistance measurement circuit without altering the traditional resistance measurement configuration. Because a traditional 4-wire resistance temperature measurement remains within the planned JNT implementation, the JNT system has the potential to largely avoid the licensing issues associated with deploying advanced, software-based signal processing in safety-related measurements at NPPs. The Johnson noise measurement does not alter the prompt response of the thermometer, but instead provides a gradual correction to the RTD's resistance versus temperature relationship.

JNT is a key temperature measurement technology in and near core where the high, lattice-damaging neutron flux makes all commercially available, low-uncertainty thermometry techniques unusable. The principal expense in a Johnson noise measurement system is in the cost recovery of the development expenses of learning to extract the signal from the competing noise sources. Neither the electronics to implement JNT nor the well-shielded transducer and cabling are especially expensive components. As such, well-implemented JNT has the potential to compete economically with other low-uncertainty thermometry techniques at high temperatures throughout NPPs. Further, JNT has the potential to become the preferred thermometry technique throughout the process control industry due to its robustness and low measurement uncertainty at a reasonable price.

\section{SIGNAL PROCESSING REQUIREMENTS}

The Nyquist equation describes the voltage produced by the motion of the electrons within a resistor at a given temperature. For frequencies below a few gigahertz, Equation 1 shows the relationship between the absolute temperature of a resistor $(\mathrm{T})$, its resistance $(\mathrm{R})$, the frequency band of measurement $\Delta \mathrm{f}$, and the measured mean-square noise voltage.

$$
\overline{V^{2}}=4 k_{B} T R \Delta f
$$

where $k_{B}$ represents Boltzmann's constant $\left(1.38 \times 10^{-23}\right.$ joules/Kelvin).

A direct measurement of the Johnson noise for temperature measurement has several challenges. First, the amplifier gain needs to be both known and stable. Second the amplifier passband and filtering effects of connection cabling must be well known to within the required measurement accuracy. Finally, the resistance of the sensor must be independently and accurately measured. To avoid these difficulties, early Johnson noise thermometers performed a ratio of two noise voltage measurements, one with a resistor at the measurement temperature and the other at a known temperature, switched onto a single amplifier channel. However, changing the connection of the sensor to the high-gain measurement circuit introduced noise and decreased reliability [3].

Another implementation restriction for Johnson noise thermometry in reactors is the capacitive effect of the cable connecting the sensing resistor to the first stage amplifiers. If the cable has significant capacitance, it will block the high frequency portion of the sensor noise before it reaches the measurement system. This filtering of the upper frequencies reduces the bandwidth of the Johnson noise signal. Under the temperature and radiation environment of 
inside the containment structure of an SMR, the cable capacitance will exhibit some change over time. We are presently designing the system to remain outside of the containment structure, which places a minimum input cable length of approximately $25 \mathrm{~m}$ between the RTD and the input electronics. This configuration adds a great deal of capacitance (many $\mathrm{nF}$ ) and complicates the front-end electronics design. We are currently correcting for this effect using the pilot-tone sweeping technique that measures channel gain and frequency variations.

Two additional signal-processing concepts were investigated previously[5]. In the first of these, the temperature measurement resistor is connected in parallel to two separate high input-impedance amplifiers. The output of these amplifiers is partially correlated, since each consists of the sum of a correlated noise voltage and uncorrelated amplifier noise voltage. If two Johnson noise amplifier signals, connected to the same resistance, are combined and time averaged, the correlated part of the noise will persist, but the uncorrelated amplifier noise will approach zero. Figure 1 illustrates the concept of cross-correlation; the measured voltage from one amplifier channel is Fourier transformed and correlated and with that from the other to form a cross power-spectral density (CPSD) measurement effectively eliminating the noise contribution from the amplifier electronics.

Johnson noise has a flat (white) spectral energy distribution. The shape of the powerspectral density functions displayed in Figs. 1 and 2 is a result of the combined effects of filtering out both the low and high frequencies from the noise and the frequency-dependent gain of the amplifier circuit. The low-frequency filtering is applied to eliminate the nonthermal noise generated by mechanical vibrations. These microphonic signals are limited to less than a few tens of kilohertz. The upper-frequency filtering is applied both to avoid aliasing higher frequencies into the measurement band as well as to minimize the impact of sensor-to-amplifier cable capacitance-induced restriction of high frequency signal transmission. The CPSD function has units of volts squared per hertz and expresses the power content per unit frequency of the measured noise signal. It is derived from the individual channel PSDs as shown in Fig. 1.

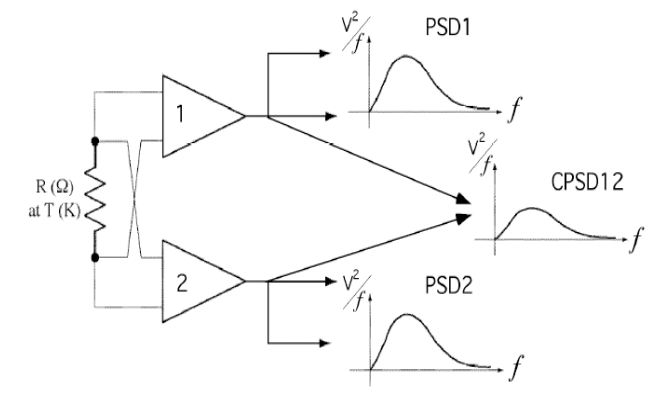

Fig. 1. Power Spectral Density function (PSD) of each amplifier channel containing both correlated and uncorrelated noise and the CPSD function from both amplifiers containing only correlated noise.

For the second concept, electromagnetic interference (EMI) spikes and microphonics are two of the biggest problems for a practical implementation of Johnson noise thermometry. In many situations, these effects can completely dominate the noise measurement. This puts a premium on well-implemented grounding, shielding, and filtering. A complementary technique to reduce these effects is to use both knowledge of the spectral energy content of Johnson noise and digital signal processing to recognize and eliminate interferences. Narrowband electromagnetic interference always appears as spikes in the long-term average CPSD that can 
be recognized and removed with only a small reduction in measurement bandwidth as illustrated in Fig. 2.

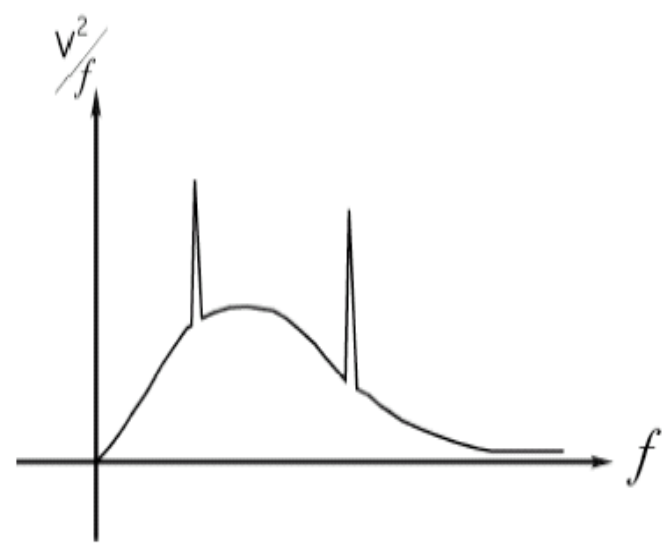

Fig. 2. CPSD with narrowband EMI Spikes.

\section{PRESENT JNT IMPLEMENTATION}

The current DOE project, led by ORNL, is focused on implementing a dual-mode resistance and Johnson noise thermometer in a rugged, integrated prototype form. The resistance measurement serves the dual purpose of providing the necessary impedance measurement for the Nyquist equation as well as providing a prompt temperature measurement. Since Johnson noise is a stochastic process, a statistically significant amount of time is required to perform a measurement. The temperature measurement in the dual-mode thermometer is therefore made as a simple resistance measurement whose resistance-totemperature conversion is quasi-continuously updated using Johnson noise. A schematic illustrating the measurement process is shown as Fig. 3. The CPSD provides the resistor noise voltage, and the resistance is independently measured, so the remaining variables that have to be known to obtain temperature from the voltage measurements are the amplifier gain as a function of frequency and the effective measurement frequency band $(\Delta f)$. The technique currently used to obtain the gains is to initially calibrate the measurement using a known temperature and treating both properties thereafter as a single constant.

A layout of the system is shown in Fig. 4. High-gain, wide-band Johnson noise preamplifiers with a precision 4-wire ohmmeter have been implemented in high-density discrete-component electronics. A continuous amplifier gain calibration scheme has also been implemented. The digital signal processing logic has been implemented in LabVIEW ${ }^{\mathrm{TM}}$ on a desktop computer utilizing a commercially-available high-speed 14-bit analog-digital converter (ADC)/data acquisition module. The preamplifier head and resistance probe have been packaged in a shielded aluminum enclosure and a coaxial signal interconnection scheme has been implemented. The preamplifier section contains two identical differential-input junction field-effect transistor (JFET) preamplifiers/filters/gain blocks, each with a voltage gain of approximately $80 \mathrm{~dB}$ and a center frequency of approximately $20 \mathrm{kHz}$. The calibration system is implemented using a swept-frequency (FM) oscillator that supplies the calibration signal over the noise bandwidth of the amplification chain by sweeping continuously between $1 \mathrm{kHz}$ to $40 \mathrm{kHz}$ and is coupled directly into the front-end preamplifier. A sub-milliamp current is 
supplied to the input also and a separate differential amplifier (ohmmeter circuit) that measures the voltage drop across the RTD. The ADCs and voltage-sweep system are remotely programmable from the LabVIEW ${ }^{\mathrm{TM}}$ software.

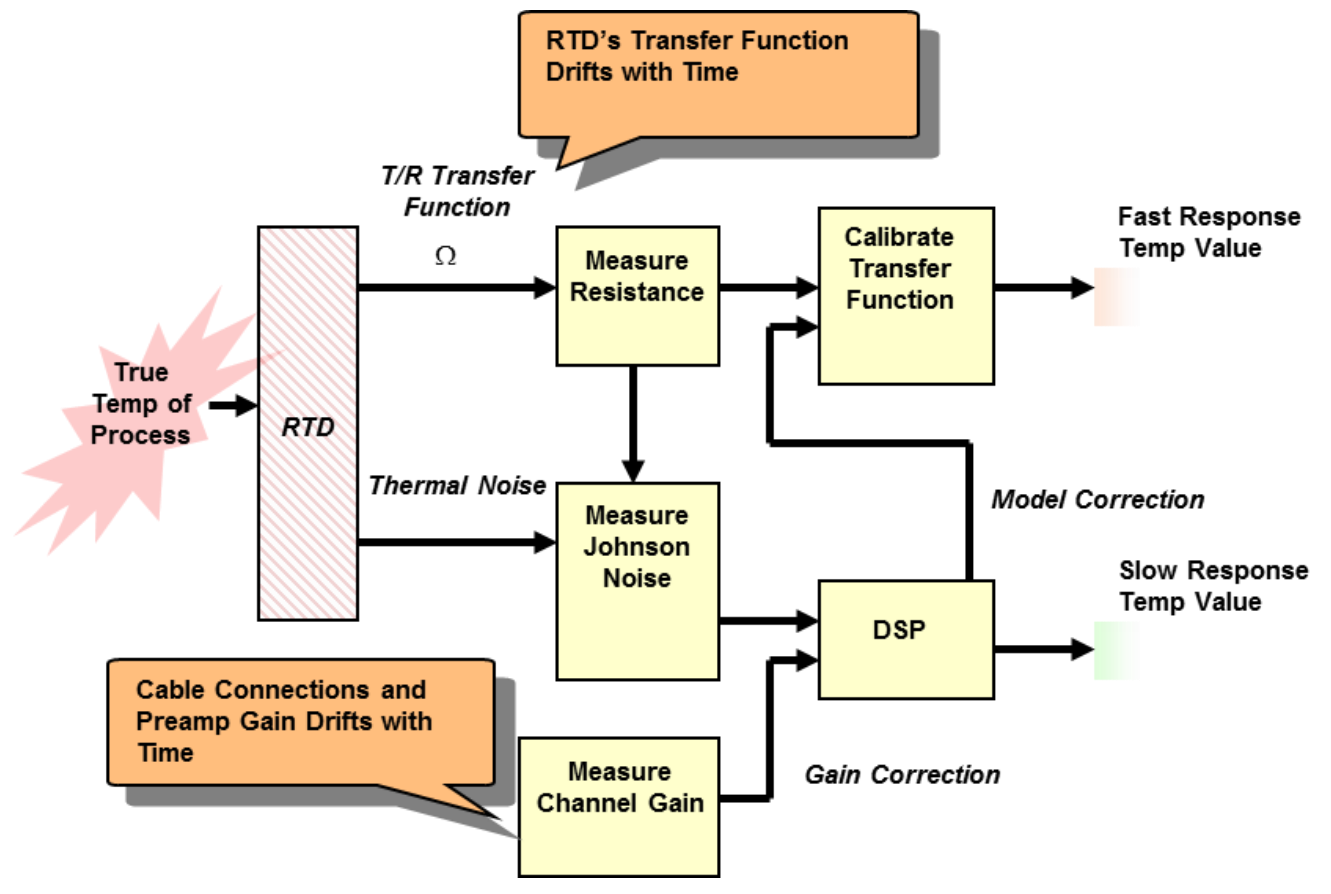

Fig. 3. Johnson Noise Thermometry measurement process schematic.

The signals processed by the data-acquisition system consist of two Johnson-noise measurement channels and one DC resistance measurement channel. The Johnson-noise channels consist of amplification followed by multiple poles of low-pass filtering. The DC resistance channel has a front-end differential receiver amplifier similar to those of the Johnson-noise channels. The signal then passes through several multi-pole high pass filters. The signals out of these three channels are then sent to a computer-based DSP using a 4 channel $2 \mathrm{M}$ sample/second ADC board.

\section{DATA}

The design of the data acquisition system and heat sources had two goals. First, they were designed to facilitate a laboratory demonstration of the JNT system. Second, they were intended to be a stable testbed for the subsequent development of the final field-deployable system. We are using three temperature generators for the measurements, which are described below:

1. A dewar for an ice bath (0 $\mathrm{C}$ measurements).

2. A tube furnace with a bang-bang controller for measurements from $200 \mathrm{C}-400 \mathrm{C}$. The tube furnace supplies a well-controlled temperature and has allowed us to calibrate the RTD against the two other RTDs, used as furnace monitors. The controller is extremely noisy and has allowed us to refine our noise rejection algorithms. 


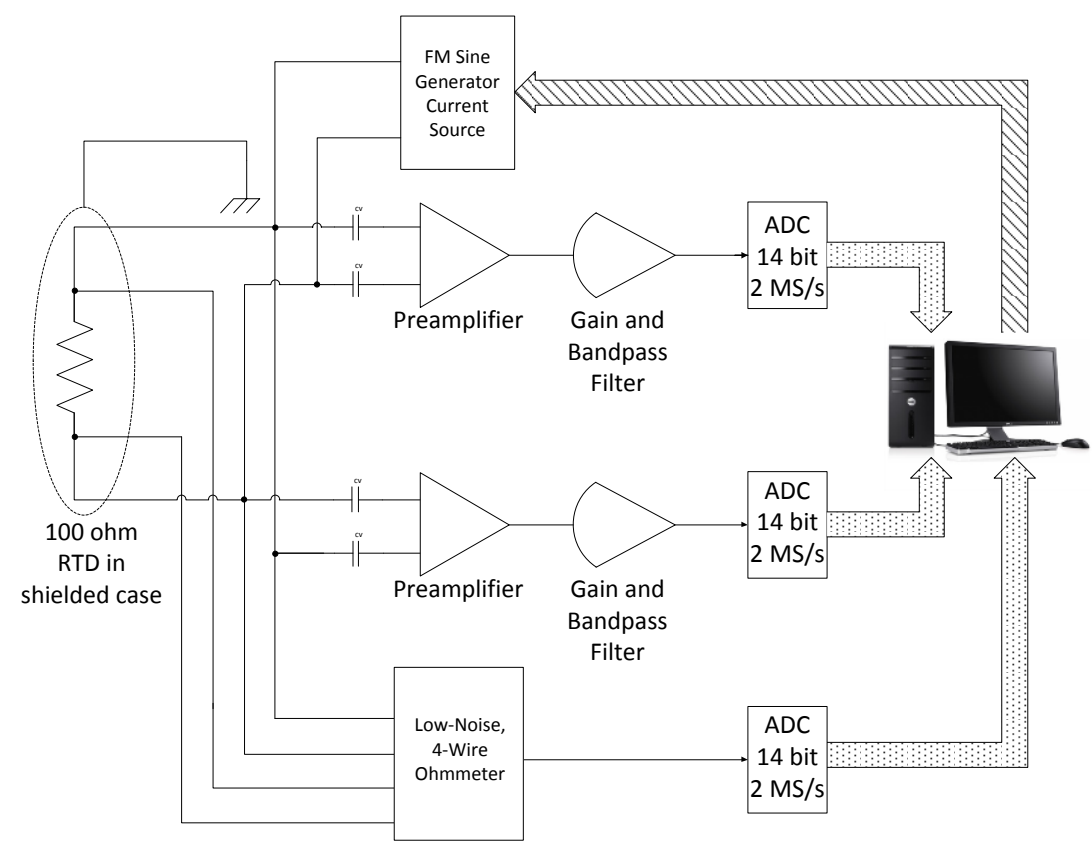

Fig. 4. Principal components and conceptual configuration of the dual-mode resistance and Johnson Noise thermometer incorporating the cross-correlation technique, digital-signal processing, and channel gain calibration.

3. An insulated heater-tape-based system that is run from a variable transformer. The system, although uncontrolled, is extremely quiet and has allowed us to compare data from the tube furnace.

There have been extensive hours of experimental measurement runs to troubleshoot the system and its various components. That data will not be presented here since it was exploratory in nature and revealed issues that we have addressed. The data presented is of the current system and a series of runs taken over several days. Calibration of the system was based primarily on two sources. First, the ice bath was used for the $0^{\circ} \mathrm{C}$ calibration. This was found to be a very stable, repeatable reference. Second, the RTDs in the tube furnace were calibrated to $\pm 0.2 \mathrm{C}$. The RTD actually utilized for the JNT system was calibrated against these. The RTD was found to read $1 \mathrm{C}$ high on the JNT system, using the JNT ohmmeter when compared to the reference RTDs. This has been incorporated into the data in Table 1. The noise-rejection algorithms appear to be working well enough so that there is no appreciable difference in data from the quiet versus noisy heaters. A screen shot from the software is shown in Fig. 5.

The results of the data are very encouraging. As can be seen, most of the runs resulted in an error of less than $1 \mathrm{~K}$. There was a single run in this set that was slightly greater than $1 \mathrm{~K}$ and the cause is not clear. We have continued to take data after this set in order to refine our JNT channel calibration. We have seen low levels of injected noise due to ground loops that are not rejected by the present software that can result in higher than expected values of temperature. This is one of the refinements we intend to implement in future versions of the software. Additionally we are trying to develop a time-efficient procedure for calibration of the system since the data is statistical and takes runs lasting several hours for reliable convergence. 
Table 1. JNT Measured Data.

Ice Bath (273 K)

\begin{tabular}{|c|c|c|c|}
\hline Run \# & $\underline{\text { JNT Temp (K) }}$ & $\underline{\text { RTD Temp (K) }}$ & Difference (K) \\
\hline 1 & 273.22 & 273.21 & 0.01 \\
\hline 8 & 273.66 & 273.17 & 0.49 \\
\hline 9 & 273.68 & 273.17 & 0.51 \\
\hline 12 & 273.07 & 273.25 & -0.18 \\
\hline
\end{tabular}

200C (473 K)

\begin{tabular}{|c|c|c|c|}
\hline Run \# & JNT Temp (K) & RTD Temp (K) & Difference (K) \\
\hline 2 & 482.54 & 482.65 & -0.11 \\
\hline 3 & 486.83 & 487.11 & -0.28 \\
\hline 4 & 489.43 & 489.46 & -0.03 \\
\hline 5 & 491.12 & 491.32 & -0.20 \\
\hline 6 & 495.68 & 495.28 & 0.20 \\
\hline 10 & 471.26 & 472.38 & -1.12 \\
\hline 11 & 472.13 & 472.39 & -0.26 \\
\hline
\end{tabular}

300C (573 K)

\begin{tabular}{|c|c|c|c|}
\hline$\underline{\text { Run \# }}$ & $\underline{\text { JNT Temp (K) }}$ & $\underline{\text { RTD Temp (K) }}$ & Difference (K) \\
\hline 7 & 597.90 & 597.53 & 0.37 \\
\hline 13 & 571.35 & 571.66 & -0.31 \\
\hline 14 & 571.40 & 572.05 & -0.65 \\
\hline 15 & 571.40 & 571.48 & -0.08 \\
\hline
\end{tabular}

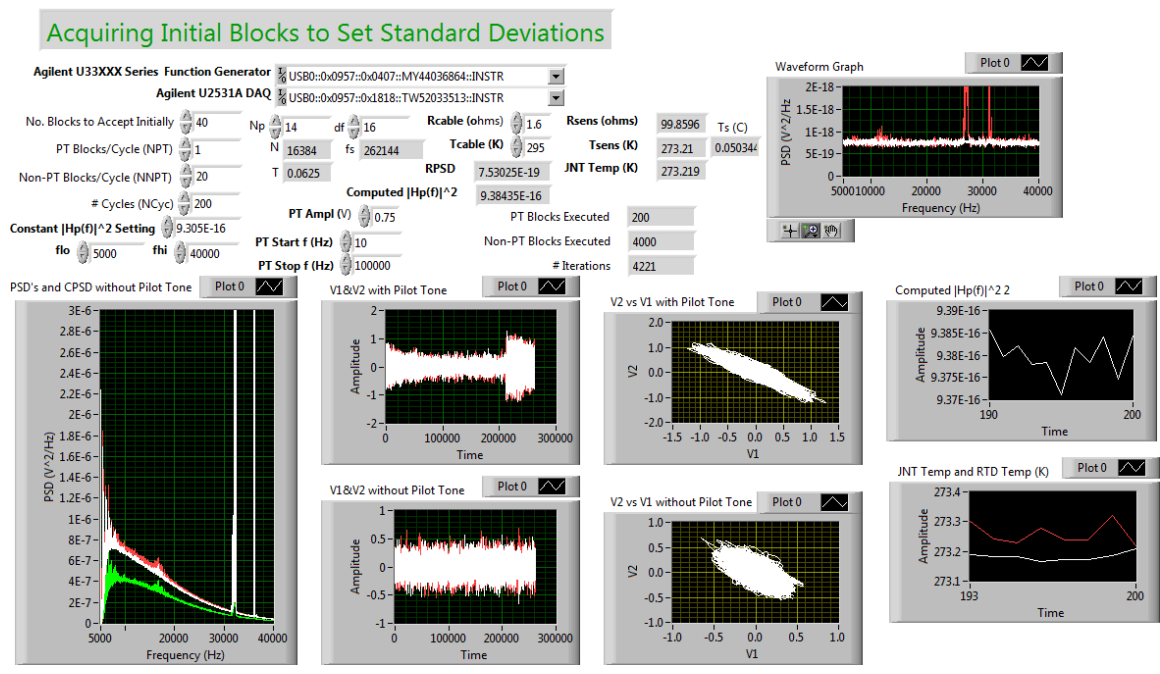

Fig. 5. Representative screen from the JNT measurement software. 


\section{DISCUSSION}

The data illustrates that the system appears to work well in the laboratory setting. The data is repeatable and appears to be accurate to within the bounds of the calibration used. Some of the issues we will address in the next phase of the project are as follows:

1. Thermal transients. We have focused on steady-state temperatures with some variation due to source temperature changes but we have not thoroughly studied the effects of transients. This will be important in field applications as the temperature will not stay constant. We have examined various approaches to data reduction for transients and will implement some set of these in the near future.

2. Cabling temperature. Because the interconnect cable from the front-end electronics to the RTD also exhibits some nonzero resistance, it too will contribute to thermal noise. This is a small value but we have compensated for this in our software for a fixed temperature. We may need some distributed temperature compensation in the future.

3. Interference noise reduction. We have gone a long way towards mitigating noise pickup but will be performing testing in the future to determine if more noise rejection is needed.

\section{SUMMARY}

Johnson-noise thermometry is becoming progressively more possible for long-term, high-reliability implementation as signal processing technology progresses and new engineering expertise is applied. While the feasibility of Johnson-noise thermometry has been demonstrated by a number of groups for nuclear reactor implementations during the past thirty years, there is currently no commercial system available for purchase. The laboratory performance of the present system indicates that further development of this system will possibly lead to commercial availability. 


\section{REFERENCES}

1. Brixy, H. G., "Temperature Measurement in Nuclear Reactors by Noise Thermometry," Nuclear Instruments and Methods, 97(1) 75-80 (November 1971)

2. Garrison, J. B. and Lawson, A. W., "An Absolute Noise Thermometer for High Temperatures and High Pressures," Review of Scientific Instruments, 20, 785-94 (1949)

3. D. E. Holcomb, R. A. Kisner, and C. L. Britton, Jr., "Fundamental Thermometry for Long-Term and High-Temperature Deployment In Generation IV Reactors," in Proceedings of the International Symposium on Future Instrumentation and Controls 2005, Tonyeong, Republic of Korea, November 1-3, 2005.

4. Plumb, Harmon H. ed., Temperature - Its Measurement and Control in Science and Industry, Part 1, Instrument Society of America, 1972, ISBN 87664-197-4.

5. R. Kisner, C.L. Britton, U. Jagadish, J.B. Wilgen, M. Roberts, T.V. Blalock, D. Holcomb, M. Bobrek, M.N. Ericson, "Johnson Noise Thermometry for Harsh Environments," Proceedings of the 2004 IEEE Aerospace Conference, March 6-14, 2004, Big Sky, MT. 